\title{
Implicações do estado nutricional em pacientes em hemodiálise: Uma revisão
}

\section{narrativa}

\author{
Implications of nutritional status in patients on hemodialysis: A narrative review \\ Implicaciones del estado nutricional en pacientes con hemodiálisis: Una revisión narrativa
}

Laís Lima de Castro Abreu

ORCID: https://orcid.org/0000-0001-6909-6774 Universidade Federal do Piauí, Brasil

E-mail: lais.castro123@ufpi.edu.br

Maria do Carmo de Carvalho e Martins ORCID: https://orcid.org/0000-0002-9107-2485 Universidade Federal do Piauí, Brasil

E-mail: carminhacmartins@yahoo.com.br

Rodrigo Feijão Rolim

ORCID: https://orcid.org/0000-0003-4863-9295 Universidade Federal do Piauí, Brasil E-mail: rodrigofeijjao2016@hotmail.com

\begin{abstract}
Resumo
A prevalência da doença renal crônica (DRC) vem aumentando consideravelmente nos últimos anos, envolve em seu curso uma série de complicações decorrentes das mudanças metabólicas e inflamatórias que originam importantes desordens nutricionais, bem como, um desequilíbrio na defesa antioxidante. A avaliação criteriosa do estado nutricional é fundamental para o planejamento de intervenções que atendam efetivamente às necessidades nutricionais dos portadores dessa patologia. Dessa forma, o presente estudo teve como objetivo identificar as principais implicações do estado nutricional relacionadas a DRC em pacientes em Hemodiálise, elencando os melhores métodos de diagnóstico nutricional e sua relação com o aumento do estresse oxidativo. Trata-se de uma revisão narrativa da literatura na qual se utilizou os descritores: Terapia Nutricional, Doença renal crônica, Hemodiálise e Estresse oxidativo. A busca foi realizada através de pesquisa com estudos dos últimos 20 anos compreendendo ao período de janeiro de 2001 a maio de 2021, nas bases de dados Pubmed, Scielo e Periódico Capes sendo utilizados os artigos originais que foram achados relevantes para o estudo e que abordassem a temática. Conforme observado, na maioria das vezes, o paciente renal apresenta-se malnutrido, com carência em reservas de vitaminas e minerais, o que diminui os mecanismos de defesa antioxidantes e favorece a instalação do estresse oxidativo renal, formando espécies reativas do metabolismo do oxigênio. Substâncias estas que são potencialmente lesivas ao organismo. Assim, pode-se afirmar que os doentes renais crônicos apresentam deficiências nutricionais importantes, sendo necessário o constante acompanhamento do estado nutricional por ser um forte preditor de morbidades e mortalidades.
\end{abstract}

Palavras-chave: Doença renal crônica; Hemodiálise; Terapia nutricional; Estresse oxidativo.

\begin{abstract}
The prevalence of chronic kidney disease (CKD) has increased considerably in recent years, involving in its course a series of complications resulting from metabolic and inflammatory changes that lead to important nutritional disorders, as well as an imbalance in the antioxidant defense. Careful assessment of nutritional status is essential for planning interventions that effectively meet the nutritional needs of patients with this pathology. Thus, the present study aimed to identify the main implications of nutritional status related to CKD in patients undergoing hemodialysis, listing the best nutritional diagnosis methods and their relationship with the increase in oxidative stress. This is a narrative review of the literature in which the following descriptors were used: Nutritional Therapy, Chronic Kidney Disease, Hemodialysis and Oxidative Stress. The search was carried out through research with studies from the last 20 years comprising the period from January 2001 to May 2021, in the Pubmed, Scielo and Capes Periodical databases, using the original articles that were found relevant to the study and that addressed the theme. As noted, most of the time, the renal patient is malnourished, with a lack of vitamin and mineral reserves, which reduces the antioxidant defense mechanisms and favors the installation of renal oxidative stress, forming reactive species of oxygen metabolism. Substances that are potentially harmful to the body. Thus, it can be stated that chronic kidney patients have significant nutritional deficiencies, requiring constant monitoring of their nutritional status as it is a strong predictor of morbidity and mortality.
\end{abstract}

Keywords: Chronic kidney disease; Hemodialysis; Nutritional therapy; Oxidative stress. 


\section{Resumen}

La prevalencia de la enfermedad renal crónica (ERC) ha aumentado considerablemente en los últimos años, implicando en su curso una serie de complicaciones derivadas de cambios metabólicos e inflamatorios que conducen a importantes trastornos nutricionales, así como un desequilibrio en la defensa antioxidante. La evaluación cuidadosa del estado nutricional es esencial para planificar intervenciones que satisfagan de manera efectiva las necesidades nutricionales de los pacientes con esta enfermedad. Así, el presente estudio tuvo como objetivo identificar las principales implicaciones del estado nutricional relacionado con la ERC en pacientes en hemodiálisis, enumerando los mejores métodos de diagnóstico nutricional y su relación con el aumento del estrés oxidativo. Se trata de una revisión narrativa de la literatura en la que se utilizaron los siguientes descriptores: Terapia Nutricional, Enfermedad Renal Crónica, Hemodiálisis y Estrés Oxidativo. La búsqueda se realizó mediante investigación con estudios de los últimos 20 años que comprenden el período de enero de 2001 a mayo de 2021, en las bases de datos Pubmed, Scielo y Capes Periodical, utilizando los artículos originales que se encontraron relevantes para el estudio y que abordaron la temática. Como se señaló, la mayoría de las veces, el paciente renal está desnutrido, con falta de reservas de vitaminas y minerales, lo que reduce los mecanismos de defensa antioxidante y favorece la instalación del estrés oxidativo renal, formando especies reactivas del metabolismo del oxígeno. Sustancias potencialmente dañinas para el organismo. Así, se puede afirmar que los pacientes renales crónicos presentan deficiencias nutricionales importantes, lo que requiere un seguimiento constante de su estado nutricional ya que es un fuerte predictor de morbilidad y mortalidade.

Palabras clave: Enfermedad renal crónica; Hemodiálisis; Terapia nutricional; Estrés oxidativo.

\section{Introdução}

A doença renal crônica (DRC) constitui-se, atualmente, em um problema de saúde pública. Trata-se da perda lenta, progressiva e irreversível das funções renais, que resulta em processos adaptativos que, por certo tempo, mantém o paciente assintomático. No entanto, quando os rins não conseguem mais remover adequadamente os produtos da degradação metabólica, o tratamento dialítico deve ser iniciado (Oliveira et al., 2012).

De acordo com Costa \& Vieira Neto \& Moysés Neto (2003), a hemodiálise (HD) é um processo de filtração do sangue que remove o excesso de líquidos, metabólitos, aminoácidos, peptídeos e vitaminas hidrossolúveis, sendo esta a terapia renal substitutiva mais utilizada. A terapia é intermitente e sua função consiste em substituir o papel dos rins, depurando o plasma sanguíneo através de um filtro conectado a um rim artificial (máquina de hemodiálise).

Em decorrência das alterações funcionais oriundas da doença e do próprio processo de hemodiálise, os pacientes frequentemente apresentam alterações no estado nutricional (Koehnlein \& Yamada \& Giannasi, 2008; Calado et al., 2009; Stefanelli et al., 2010; Oliveira et al., 2012). A desnutrição está presente em 10 a $70 \%$ dos doentes mantidos em hemodiálise e, 18 a $26 \%$ dos indivíduos sob tratamento de diálise peritoneal ambulatorial contínua apresentam algum sinal de desnutrição (Dantas et al., 2012).

A definição de desnutrição energético-proteica pode ser padronizada para pacientes com DRC com foco na etiologia e relação com inflamação em: 1) associada à doença ou condição crônica com inflamação continuada; 2) associada à doença crônica com inflamação mínima ou não percebida; 3) associada à doença ou injúria aguda com inflamação grave, e 4) associada à baixa ingestão alimentar crônica pura não relacionada à doença. É amplamente conhecido que a desnutrição tem alta prevalência e está intimamente associada a resultados clínicos adversos e aumento da taxa de hospitalização, complicações e mortalidade nessa população (Oliveira et al., 2019) A patogênese da desnutrição na DRC é multifatorial e complexa, e as principais causas são a redução da ingestão alimentar e do anabolismo de nutrientes, e a presença de hipercatabolismo (Kope $\&$ Kalantar-Zadeh).

$\mathrm{Na} \mathrm{DRC}$, a perda de funções relacionadas com a manutenção do equilíbrio hidroeletrolítico e ácido-base, com a função reguladora hormonal, e com a excreção de catabólitos desencadeiam sérios problemas para o paciente, tais como anemia, deficiência imunológica, tendência a hemorragias, desordens no metabolismo de lipídios, carboidratos e proteínas, além de vários distúrbios resultantes das toxinas presentes no plasma ou outros fluidos corporais (Bastos et al., 2010).

Trabalhos têm demonstrado que algumas manifestações clínicas dessa doença podem ser decorrentes da 
superprodução de espécies reativas de oxigênio, em função do desequilíbrio entre substâncias pró-oxidantes e antioxidantes, favorecendo a manifestação do estresse oxidativo, que contribui para a mutagênese, peroxidação lipídica (PL), oxidação de proteínas, envelhecimento e doenças crônicas (Bianchi et al., 2009; Knap et al., 2009). A uremia tem sido considerada como um estado pró-oxidante marcado por aumento de lipoperoxidação e diminuição da atividade antioxidante (Kosch et al., 2003). Porém, não está clara a natureza desse estresse oxidativo e se o mesmo é exacerbado pela diálise (Vaziri et al., 2004). A lipoperoxidação tem sido associada à patogênese de doenças comumente encontradas em indivíduos que realizam HD, como a arteriosclerose (Nihi et al, 2010).

O estado nutricional de doentes renais em HD tem sido estudo de interesse por sua relação direta com a morbimortalidade (Dantas et al., 2012). O aporte de nutrientes é essencial na manutenção do balanço nitrogenado positivo, melhorando os resultados do tratamento dialítico e a qualidade de vida de doentes renais crônicos (Cuppari \& Kamimura, 2009).

Ainda há controvérsias quanto ao método de avaliação nutricional mais eficaz para diagnosticar a desnutrição calórico-proteica em pacientes em HD. A maioria dos métodos apresenta limitações na sua aplicação, tendo em vista que alguns fatores relacionados com a doença podem afetar a validade das técnicas utilizadas (Calado et al., 2007; Koehnlein \& Yamada \& Giannasi, 2008). Dentre os métodos de avaliação nutricional destacam-se a antropometria, que avalia o grau de adiposidade e de massa magra através de medidas de estatura, peso corporal, cálculo do índice de massa corporal (IMC), entre outras; parâmetros bioquímicos, dos quais a albumina sérica tem sido o mais utilizado para a avaliação do estado nutricional de pacientes em hemodiálise; além de métodos de avaliação subjetivos (Santos et al, 2004).

O conhecimento e caracterização do estado nutricional de doentes em HD são fundamentais tanto para a prevenção da desnutrição quanto para intervir apropriadamente nos doentes que já se apresentam desnutridos, contribuindo, dessa forma, para um atendimento de melhor qualidade. Estudos adicionais nessa linha de pesquisa são importantes para identificar os métodos mais confiáveis de identificação precoce de doentes em HD em situação de risco nutricional, o que justifica a importância do presente trabalho.

Nesse sentido, o estudo teve como objetivo identificar as principais implicações do estado nutricional em pacientes em hemodiálise, elencando os melhores métodos de diagnóstico nutricional nessa situação e sua relação com o aumento do estresse oxidativo.

\section{Metodologia}

Trata-se de uma revisão de literatura de caráter narrativo, na qual realizou-se uma pesquisa bibliográfica durantes os messe de janeiro a maio de 2021 nas bases de dados PubMed, Scielo e portal periódicos CAPES, através da busca dos termos descritos no DeSC: Terapia Nutricional, Doença renal crônica, Hemodiálise e Estresse oxidativo, em português, inglês e espanhol. Os critérios de inclusão para o estudo foram que os trabalhos fossem estudos originais publicados no período de janeiro de 2001 a maio de 2021, que tratassem do tema em questão, abordando as principais implicações do estado nutricional em pacientes em hemodiálise, métodos de diagnóstico nutricional e sua relação com o aumento do estresse oxidativo nessa patologia. Para a seleção dos artigos a serem utilizados nesse estudo inicialmente foi feita a leitura dos resumos e rejeitaram-se aqueles que não preencheram os critérios de inclusão.

\section{Resultados e Discussão}

\subsection{Implicações nutricionais da doença renal crônica}

Doentes renais crônicos sofrem com frequência de anormalidades nutricionais e a redução da função renal contribui para o aparecimento de uma série de distúrbios hidroeletrolíticos, hormonais e metabólicos que, direta ou indiretamente, 
contribuem para o desenvolvimento de estado nutricional adverso, marcado pela depleção de reservas de gordura e proteínas, especialmente do tecido muscular. Contudo, na última década, tem sido observado aumento da prevalência de sobrepeso e obesidade em doentes renais crônicos (Koehnlein \& Yamada \& Giannasi, 2008).

A desnutrição proteico-energética é um achado frequente em doentes renais crônicos em diálise e está associada à perda de peso, à depleção energética e ao hipercatabolismo proteico. A prevalência da desnutrição nessa população é elevada e, dependendo do parâmetro utilizado, pode variar de $10 \%$ a $70 \%$. Sua etiologia é multifatorial, sendo a ingestão alimentar insuficiente, o catabolismo aumentado, as alterações hormonais, a inflamação e as doenças associadas os principais fatores causais (Calado et al., 2009).

De acordo com as recomendações de nutrição da National Kidney Foundation/ Kidney Disease Outcome Quality Initiative (NFK/KDOQI), publicadas em 2006, a desnutrição proteico-energética é caracterizada pela perda insidiosa de gordura corporal, de reservas somáticas proteicas, redução da concentração das proteínas séricas e diminuição da capacidade funcional.

No Consenso Europeu organizado pela European Dialysis and Transplant Nurses Association (EDTNA) e European Renal Care Association (ERCA) em 2002, a desnutrição de doentes em HD foi definida como "má nutrição proteico-energética com redução da massa gorda e da massa magra", sendo ainda classificada em dois tipos. Desnutrição tipo 1 resulta da ingestão energética e/ou proteica inadequada, e é caracterizado por concentração sérica de albumina preservada ou ligeiramente diminuída, catabolismo proteico aumentado, gasto energético em repouso normal, resposta inflamatória ausente e possível aumento do estresse oxidativo. Esse tipo de desnutrição está associada à uremia per se, à inatividade física, à subdiálise e/ou fatores psicossociais, podendo ser revertida facilmente, quando otimizadas as terapêuticas médicas e nutricionais. Desnutrição tipo 2, por sua vez, está associado à ativação da resposta inflamatória sistêmica, marcada por aumento da proteína C reativa e de citocinas, em que a hipoalbuminemia, o aumento do catabolismo proteico e do metabolismo basal, a anorexia e aumento do estresse oxidativo são características associadas. Os doentes com esse tipo de desnutrição apresentam muitas vezes várias comorbidades associadas. É importante destacar que os dois tipos de desnutrição podem coexistir clinicamente.

Segundo Stefanelli et al. (2010), vários fatores predispõem os doentes renais crônicos em HD à desnutrição energético-proteica, entre eles destacam-se: anorexia resultante do acúmulo de metabólitos tóxicos; acidose metabólica; resistência à ação de hormônios anabólicos; e presença de comorbidades associadas, tais como diabetes mellitus (DM), hipertensão arterial sistêmica (HAS) e insuficiência cardíaca congestiva. Além disso, quantidades significativas de peptídeos, aminoácidos e outros nutrientes são perdidos a cada sessão de diálise, o que contribui para o aparecimento de quadros de subnutrição.

Fatores como resistência insulínica, circulação de mediadores inflamatórios, acidose, aumento da secreção de hormônios catabólicos e oferta inadequada de substratos nutricionais estão entre as principais causas de hipercatabolismo nesses doentes, contribuindo para a perda acentuada de massa magra através da ativação de catabolismo proteico muscular, neoglicogênese e alteração do metabolismo de aminoácidos (Berbel et al., 2011).

O fornecimento adequado de nutrientes nos diversos estágios do tratamento é fundamental para manutenção e recuperação do estado nutricional, visto que a ingestão dietética insuficiente nessa população é considerada uma das maiores causas da desnutrição energético-proteica, levando a uma redução das reservas de gordura e massa magra corporal. A uremia, os processos inflamatórios e infecciosos frequentes também comprometem o estado clínico e nutricional, refletindo na perda de peso seco (Leal et al., 2008).

Além da importância clínica do déficit energético-proteico, nos doentes em HD, a adequação de água e de minerais ingeridos pelos doentes é de extrema relevância, principalmente em relação ao cálcio, ferro, sódio, potássio e fósforo. Tais nutrientes estão relacionados a complicações, uma vez que o rim não consegue mais manter o controle do meio interno 
(Koehnlein \& Yamada \& Giannasi, 2008).

Com relação às vitaminas, pequenas perdas de vitaminas hidrossolúveis ocorrem durante a HD, e alguns fatores são responsáveis por essas perdas, incluindo sua degradação pelos rins ou a produção diminuída, as perdas através da diálise, a interferência de medicamentos na absorção, excreção e metabolismo das vitaminas, e a ingestão alimentar insuficiente causada pela anorexia (Ribeiro-Junior et al., 2017).

De acordo com Chaves et al. (2007), alterações ósseas importantes são observadas, sendo uma delas a osteomalácia, caracterizada por um grave defeito da mineralização óssea, podendo ser resultante da lentidão acentuada da deposição de cálcio na matriz óssea. Os autores ainda citam que o perfil lipídico pode apresentar-se tipicamente trombogênico, com elevações dos níveis de triglicérides e reduções do HDL-colesterol (HDL-c). Outra grave consequência da DRC é a anemia, causada, principalmente, pela produção insuficiente de EPO (Abensur, 2010).

Apesar da elevada prevalência de desnutrição energético-proteica em doentes renais crônicos, sobrepeso e a obesidade também são distúrbios nutricionais presentes nessa população. Estudos têm sugerido que a adiposidade pode estar associada ao desenvolvimento de DRC, sendo o DM tipo 2 e a HAS as duas principais causas de DRC, também associadas com obesidade (Mafra \& Forage, 2006; Paula et al., 2006).

De acordo com Stratta et al. (2007), o principal aspecto negativo da obesidade na DRC é sua relação com a doença cardiovascular. Como já mencionado, a doença cardiovascular é a principal causa de morbimortalidade nos doentes renais crônicos. A obesidade, por sua vez, é um fator de risco nutricional para o desenvolvimento dessa comorbidade, particularmente a obesidade central, caracterizada por acúmulo de gordura visceral.

Para Mafra \& Forage (2006), a obesidade cursa com alterações hemodinâmicas renais caracterizadas por aumento do fluxo plasmático renal, hiperfiltração glomerular e em alguns casos retenção salina. Além disso, a obesidade pode acelerar a evolução de glomerulopatias pré-existentes e pode induzir lesão renal de novo em doentes uninefrectomizados. Finalmente, a obesidade tem sido associada a um tipo específico de lesão glomerular, manifestada por proteinúria, glomerulomegalia e esclerose glomerular segmentar e focal, o que também contribui para a gênese da DRC (Stratta et al., 2007).

Apesar dos aspectos negativos da obesidade, como fator de risco para DRC e outras doenças e agravos crônicos não transmissíveis, há uma série de trabalhos demonstrando que a sobrevida de doentes em HD aumenta conforme o IMC aumenta, conferindo a esse aumento relativo de peso um caráter protetor. Esse fenômeno foi denominado de epidemiologia reversa da obesidade (Mafra \& Forage, 2006; Paula et al., 2006; Stratta et al., 2007). Contudo, alguns autores postulam que a vantagem de sobrevida de doentes com peso acima do normal é limitada para aqueles com massa muscular normal ou elevada (Calado et al., 2009).

Segundo Cabral et al. (2005), os fatores envolvidos nessa relação ainda não foram totalmente elucidados, mas a gordura corpórea poderia exercer um efeito protetor, suprindo as necessidades energéticas e poupando a massa corpórea magra em situações clínicas desfavoráveis, tais como infecções e/ou inflamação, situações que acometem com frequência os doentes renais crônicos.

Diante desses fatos, o estado nutricional dos doentes em HD tem despertado grande interesse de estudos, justificado por sua relação direta com a morbidade e mortalidade. Conhecer o estado nutricional de uma população em HD é imprescindível para uma intervenção precoce e para prevenir distúrbios nutricionais, contribuindo para um atendimento nutricional de melhor qualidade. Todo paciente em HD deve ser avaliado no início do tratamento e reavaliado a cada seis meses. Por meio da avaliação do estado nutricional é possível identificar indivíduos acima do peso, desnutridos ou em risco nutricional e, a partir dos resultados, propor uma conduta dietética eficaz a fim de promover um bom padrão nutricional a esses doentes. 


\subsection{Avaliação do estado nutricional de doentes renais crônicos}

As recomendações da NKF/KDOQI de nutrição (2006) recomendam a avaliação nutricional frequente de todos os doentes em HD, e referem que nenhum indicador nutricional ou método de avaliação isoladamente pode ser considerado como "padrão". E, adicionalmente, aconselham a utilização simultânea de pelo menos dois dos vários métodos propostos.

Segundo Oliveira et al. (2010a) os métodos de avaliação do estado nutricional para doentes renais podem ser subjetivos (história clínica e exame físico nutricional) e objetivos (antropometria, exames bioquímicos, consumo alimentar e bioimpedância elétrica). Para Cabral et al. (2005) os parâmetros de avaliação do estado nutricional e as interpretações apropriadas constituem um grande desafio em se tratando de doentes renais crônicos, devido ao fato de serem influenciados por muitos fatores não nutricionais.

\subsubsection{Antropometria clássica e avaliação do estado nutricional de doentes renais crônicos}

A antropometria é um método de investigação científica em nutrição que se ocupa da medição das variações nas dimensões físicas e na composição global humana em diferentes idades e em distintos graus de nutrição ou estados nutricionais (Koehnlein \& Yamada \& Giannasi, 2008; Dantas et al., 2012). Ela é utilizada nas clínicas de hemodiálise por ser um método simples, seguro, prático, validado, de baixo custo e clinicamente útil na avaliação do estado nutricional de doentes renais crônicos. Também permite avaliar o grau de adiposidade e de massa magra do paciente, e abrangem medidas de estatura, peso corporal, percentual do peso atual em relação ao ideal, espessura das pregas cutâneas, circunferência do braço, área muscular do braço, além de permitir o cálculo de índices utilizados na avaliação do estado nutricional, como o IMC (Oliveira et al., 2010a).

O peso corporal é um importante parâmetro nutricional, sendo indicador básico e importante na prática clínica, devido a sua determinação simples, precisa e pela boa aceitação por parte dos pacientes. Entretanto, a utilização do peso apresenta limitações, pois, mede todos os compartimentos corporais de uma só vez, não especificando qual compartimento está alterado (Passoni, 2005). Para minimizar suas limitações deve-se considerar na avaliação do estado nutricional: o peso usual (PU), que é informado pelo próprio paciente; o peso ideal (PI), obtido por tabelas específicas ou fórmulas; e a perda de peso não intencional por certo período, que é um importante parâmetro para avaliar a desnutrição, pois perdas ponderais graves estão associadas à maior morbimortalidade (NKF/KDOQI, 2006).

O IMC, calculado pela razão entre o peso corporal e o quadrado da estatura, é utilizado para estimar o nível de adiposidade em indivíduos de grupos etários diferentes, no qual, valores inferiores a 18,5 kg/m2 devem ser considerados como importante sinal de desnutrição energético-proteica em adultos (Favalessa et al., 2009). É recomendável que a interpretação dos valores de IMC seja feita em associação com outros marcadores nutricionais, principalmente nos valores mais elevados, visto que os valores reduzidos de IMC estão claramente associados com maior taxa de mortalidade (Koehnlein \& Yamada \& Giannasi, 2008; Calado et al., 2009; Stefanelli et al., 2010).

A medida de pregas cutâneas constitui o meio mais conveniente para estabelecer indiretamente a massa corpórea de gordura, e apesar de suas limitações, as medidas das pregas cutâneas podem ser úteis para avaliar em longo prazo as reservas de tecido adiposo subcutâneo em pessoas com doenças crônicas. A determinação de mudanças agudas na composição corpórea utilizando tais medidas não pode ser feita com confiança. Esta limitação do método da determinação das pregas cutâneas ocorre pelo fato de que a redistribuição corpórea de fluidos pode causar edemas, como aqueles que ocorrem na insuficiência renal (Cabral et al., 2005).

As medidas de circunferências corporais são de fácil execução, podendo ser consideradas isoladamente ou em combinação com as pregas cutâneas para classificar o estado nutricional e a distribuição de gordura corporal. A circunferência do braço (CB), por exemplo, representa a somatória dos tecidos ósseo, muscular e gorduroso do braço, refletindo a composição 
corporal, sem distinção entre gordura e massa magra. Sua utilização em combinação com a prega cutânea tricipital (PCT) possibilita o cálculo da circunferência muscular do braço (CMB), uma medida indireta de massa muscular (Passoni, 2005).

Outra medida de circunferência utilizada na avaliação antropométrica do estado nutricional é a circunferência da cintura (CC), que se constitui em uma ferramenta importante para identificar o grau de adiposidade central em indivíduos que apresentam risco de desenvolver complicações metabólicas e cardiovasculares. A CC é utilizada em estudos epidemiológicos devido a sua facilidade de medida e também por apresentar uma associação consistente e geralmente forte com outros fatores de risco para o desenvolvimento de doenças cardiovasculares (Cesarino et al, 2013). Ela é um dos indicadores mais utilizados na aferição da distribuição centralizada do tecido adiposo em avaliações individuais e coletivas. Contudo, as diferenças na composição corporal dos diversos grupos etários e raciais dificultam o estabelecimento de pontos de corte universais para classificação de adiposidade central (Cabral et al., 2005).

Para Oliveira et al. (2010a), um fato que torna questionável a utilização de medidas antropométricas de doentes em diálise é a falta de normas referenciais que levem em conta o sexo, a idade e a raça. Além disso, a antropometria não identifica alterações nutricionais em períodos curtos de tempo, nem a deficiência específica de um nutriente (Cuppari; Kamimura, 2009).

\subsubsection{Marcadores bioquímicos utilizados na avaliação do estado nutricional de doentes renais crônicos}

Os marcadores bioquímicos fornecem medidas objetivas das alterações do estado nutricional, com a vantagem de possibilitar seguimento ao longo do tempo e a realização de intervenções nutricionais. Os exames laboratoriais são de grande valia na identificação de alterações nutricionais, porém vários fatores podem interferir e limitar a interpretação dos dados obtidos. Neste sentido, testes únicos e isolados, sem considerar outros parâmetros de avaliação nutricional e também a idade, sexo, estado fisiológico, condições ambientais e fatores não nutricionais, tais como drogas, estresse e injúria, podem dificultar ou impossibilitar a interpretação dos resultados obtidos (Dantas et al., 2012).

Entre os indicadores bioquímicos das reservas proteicas mais comumente empregados na avaliação nutricional de doentes renais crônicos, destacam-se aqueles relacionados com as reservas de proteínas viscerais, tais como os níveis séricos de albumina, pré-albumina e transferrina. Apesar dessas proteínas serem sensíveis à diminuição crônica da ingestão proteica e energética, elas também são significativamente afetadas pelos vários distúrbios associados à DRC. Dentre as proteínas viscerais, a albumina continua sendo o marcador bioquímico das reservas proteicas mais empregado, tanto na pesquisa quanto na prática clínica (Cuppari \& Kamimura, 2009). Segundo Kubrusly et al. (2012), a utilização da albumina como marcador bioquímico deve-se à facilidade de sua medição e a sua associação com eventos clínicos nessa população, uma vez que estudos têm mostrado uma forte correlação entre os baixos níveis de albumina e o aumento do risco de morbimortalidade (Mazairac et al., 2011; Schmidt et al., 2012).

Embora a albumina seja um marcador de alta especificidade, sua sensibilidade no diagnóstico da desnutrição é baixa, pois seus níveis podem ser influenciados por diversos fatores, incluindo o estado de hidratação, perdas externas e pela redução de sua síntese. E, considerando que as concentrações séricas de albumina podem reduzir rapidamente em resposta ao estresse ou inflamação, sua concentração pode não refletir o estado nutricional de doentes com enfermidades agudas (Herselman et al., 2010).

Apesar das limitações apontadas acima, a albumina destaca-se por ser um potente preditor de morbidade e mortalidade na DRC, independentemente das causas que levam à redução da sua concentração sérica. De fato, particularmente na população de doentes em diálise, valores de albumina sérica inferiores a 2,5 g/dl são associados a um risco de morte 20 vezes maior quando comparados aos valores de referência de 4,0 a 4,5 g/dl; e mesmo valores considerados dentro da faixa de normalidade (3,5 e 4,0 g/dl) estão associados com um risco duas vezes maior (Cuppari \& Kamimura, 2009). Assim, tem sido recomendado que a concentração sérica de albumina na população de doentes renais crônicos seja mantida acima de 4,0 g/dl 
(Kubrusly et al., 2012).

Outro marcador bioquímico do estado nutricional é a ureia sérica, que pode ser útil na monitoração da ingestão proteica atual do paciente. A ureia é produto final do metabolismo proteico, possuindo, portanto, relação direta com a ingestão ou com a quebra endógena de proteínas, ou combinação de ambas, além de ser dependente da função renal e intensidade da diálise em indivíduos com DRC. A partir de sua cinética, pode-se calcular a ingestão proteica atual do paciente e verificar a sua adequação em relação à quantidade recomendada, porém, caso a ingestão proteica não seja considerada frente a uma redução dos níveis de ureia, isso poderá determinar uma redução da prescrição de diálise, o que pode resultar em agravamento do estado nutricional devido à uremia. Além disso, níveis baixos de ureia pré-HD estão correlacionados com o aumento da mortalidade (Draczevski; Teixeira, 2011; Cuppari \& Kamimura, 2009).

Durante a hemodiálise, o excesso de ureia é retirado, mas essa eliminação não é total, portanto, para não haver acúmulo, é muito importante equilibrar a quantidade de proteínas consumida e a de ureia produzida, sendo que durante a diálise a ureia é rapidamente depurada em relação à creatinina, tendo seus níveis altamente diminuídos após a realização de sessão de HD (Costa \& Vieira Neto \& Moysés Neto, 2003).

A creatinina sérica é também considerada um marcador nutricional importante, uma vez que níveis abaixo dos esperados (<10 mg/dl) refletem na diminuição da massa muscular e tem sido associado com maior taxa de mortalidade nos doentes em HD (Dantas et al., 2012). Os níveis séricos de creatinina pré-diálise são proporcionais à ingestão de carne e à massa magra corporal em pacientes em HD com pouca ou nenhuma função renal. Assim, em doentes com creatinina prédiálise muito reduzida ou com diminuição da creatinina ao longo do tempo, deve-se suspeitar de redução da massa muscular. Nessa situação, outros parâmetros nutricionais devem ser investigados, particularmente os relacionados ao apetite e à ingestão de energia e proteína (Cuppari \& Kamimura, 2009).

As vantagens do uso da ureia e da creatinina são que elas são facilmente disponíveis e de baixo custo, além de refletirem a ingestão alimentar recente da maioria dos doentes. No entanto, várias condições interferem na acurácia dessas medidas, como erros na coleta de sangue, ingestão de carnes, atividade física intensa e uso de diuréticos (Draczevski \& Teixeira, 2011).

\subsubsection{Uso da bioimpedância elétrica (BIA) na avaliação nutricional de doentes renais crônicos}

Dentre os métodos de avaliação nutricional de fácil aplicabilidade na prática clínica, a bioimpedância elétrica (BIA) tem sido amplamente aceita pela comunidade de nefrologistas por fornecer uma estimativa dos compartimentos de massa magra e de gordura corporal (Cuppari \& Kamimura, 2009). Ela destaca-se entre os vários métodos disponíveis para avaliação da composição por se tratar de uma técnica simples, rápida, segura, além de apresentar boa reprodutibilidade e não oferecer riscos, sendo, por essas razões, cada vez mais utilizada na prática clínica (Gómez, 2011).

Esse método baseia-se no princípio de que os tecidos corporais oferecem diferentes oposições à passagem da corrente elétrica. Os tecidos magros são altamente condutores de corrente elétrica devido a grande quantidade de água e eletrólitos, apresentando baixa resistência à passagem da corrente elétrica. Por outro lado, a gordura, o osso e a pele constituem um meio de baixa condutividade e elevada resistência (Kyle et al., 2004; Eickemberg et al, 2011). Essa oposição, chamada impedância elétrica $(Z)$, é formada por dois vetores, a resistência elétrica $(\mathrm{R})$ e a reatância elétrica $(\mathrm{X})$, em que R indica a oposição do fluxo de corrente elétrica através do corpo e é inversamente proporcional a quantidade de fluidos, associando-se às características antropométricas do indivíduo (Mushnick, 2003; Piccoli, 2002). Já a X consiste na oposição ao fluxo da corrente causada pela capacitância produzida pelas membranas celulares, sendo um indicador da quantidade de massa celular corporal ou massa magra, relacionada com a estrutura e a função de membranas celulares (Kyle et al, 2004).

A massa corporal pode ser dividida nos compartimentos massa gorda e massa magra, sendo a massa corporal magra 
subdividida em um modelo multicompartimental composto de: esqueleto; tegumento; músculo esquelético e órgãos viscerais; e água corporal total, distribuída nos compartimentos intra e extracelular. A partir da massa magra corporal sem a massa mineral óssea ou água extracelular têm-se a massa celular corporal (MCC), cuja medida é um marcador de depósitos proteicos viscerais e somáticos combinados, sendo considerado o compartimento corporal mais ativo metabolicamente (Oliveira et al., 2010b).

Conforme Talluri et al. (2003), a MCC é um bom preditor de estado nutricional do indivíduo, pois é mais sensível a alterações no estado de proteína e tecido magro em comparação ao IMC. Além disso, a depleção de massa muscular em certas condições patológicas pode ser mais bem descrita pela perda de MCC (por exemplo, sarcopenia, anorexia nervosa e pacientes em diálise).

Assim como a MCC, o ângulo de fase (AF) pode ser obtido por meio da relação entre medidas diretas da R e da X, sendo calculado diretamente pela equação X/R x 180\% (Barbosa-Silva et al., 2003). Segundo Eickemberg et al. (2011), o AF é dependente da capacitância dos tecidos e está associado com a qualidade, tamanho e integridade celular. Trata-se de uma ferramenta de diagnóstico nutricional cada vez mais utilizada na prática clínica. Estudos recentes têm validado esse ângulo como indicador prognóstico em doentes críticos. É importante destacar que o AF, relacionado ao equilíbrio celular, tem sido utilizado como medida de gravidade de doença, como instrumento de avaliação funcional e como indicador geral de saúde (Barbosa-Silva et al., 2005; Gupta et al., 2004 e 2009).

Pesquisas realizadas para investigar o papel do AF como indicador de prognóstico em pacientes com doenças graves (como DRC, alguns tipos de câncer, Síndrome de Imunodeficiência Adquirida - AIDS, doença pulmonar obstrutiva crônica, cirrose hepática) e em pacientes criticamente doentes, evidenciaram associação positiva entre os valores do AF e o tempo de sobrevida dos pacientes. Dessa forma, o AF poder ser uma importante ferramenta para avaliar sinais clínicos e monitorar a progressão da doença, sendo superior a outros indicadores do estado nutricional, como marcadores bioquímicos ou antropométricos (Gupta et al., 2004 e 2009; Barbosa-Silva et al., 2005; Azevedo et al., 2007; De Luis et al., 2007).

De acordo com Gómez (2011), o uso da BIA tem demonstrado eficiência na aferição dos compartimentos corporais em diversas situações clínicas, incluindo desnutrição, traumas, estados pré e pós-operatório, doenças hepáticas e insuficiência renal; e também em situações especiais do desenvolvimento humano como gestação e infância. De fato, embora a BIA seja um método de avaliação da composição corporal altamente aceito pela comunidade científica, seus resultados podem ser afetados por fatores como a alimentação, o exercício físico e a inadequada ingestão de líquidos em períodos que antecedem a avaliação (estados de desidratação ou retenção hídrica), utilização de diuréticos e ciclo menstrual.

Em doentes renais crônicos, alguns autores não recomendam a utilização da BIA na mensuração das variações agudas de fluido corporal de doentes em diálise, e referem-se que este método seria útil em estudos de seguimento e não comparativos (Jambassi Filho et al., 2010; Kyle et al., 2004). Contrariamente, outros estudiosos defendem a sua aplicação na avaliação das mudanças hídricas agudas da diálise (Barbosa-Silva et al., 2003; Barreto-Silva et al., 2008).

A principal limitação para utilização da BIA é o estado de hidratação, uma vez que na hiper-hidratação ocorre superestimação da massa magra. Uma desvantagem adicional do método é que a massa corporal magra é estimada a partir de equações preditivas que foram desenvolvidas para população saudável, com volemia estável; e, no caso dos doentes renais, a volemia é constantemente alterada, tornando essas equações imprecisas (Pupim et al, 2002).

Alguns autores acreditam no potencial dos marcadores da BIA para detectar alterações precoces no estado nutricional (mesmo antes de alterações no IMC e em marcadores bioquímicos), e enfatizam a necessidade de realização de estudos longitudinais em maior número de pacientes em diferentes populações para a confirmação desses resultados, e com vista à indicação posterior desse exame no seguimento nutricional dos pacientes em hemodiálise (Barreto-Silva et al., 2008; Oliveira et al., 2010b).

A pequena utilização da BIA na prática clínica e em situações epidemiológicas deve-se à carência de valores de 
referência para a população em geral. Destaca-se que tais valores são necessários para avaliar corretamente desvios individuais em relação à média populacional e para comparar estudos, sendo recomendado o estabelecimento de critérios mais confiáveis para análise e interpretação dos resultados (Barbosa-Silva et al., 2003; Kyle et al., 2004; Eickemberg et al., 2011).

\subsection{Estresse oxidativo na doença renal crônica (DRC)}

O estresse oxidativo pode ser definido como o desequilíbrio entre os níveis de compostos pró-oxidantes e de antioxidantes, com predomínio dos primeiros. O estado pró-oxidante vem sendo relacionado a várias doenças, como doenças cardiovasculares, neurodegenerativas, autoimunes, renais e cânceres, ora como sua causa, e em alguns casos como consequência (Vellosa et al., 2013).

Os antioxidantes podem ser classificados em enzimáticos e não enzimáticos. Os principais componentes do sistema antioxidante enzimático são as enzimas superóxido dismutase (SOD), catalase (CAT) e glutationa peroxidase (GPx), que agem no início da cadeia de formação das espécies reativas, evitando o acúmulo do ânion superóxido (O2-) e de peróxido de hidrogênio (H2O2). Os antioxidantes não enzimáticos incluem compostos produzidos in vivo, tais como a glutationa reduzida (GSH), a ubiquinona, o ácido úrico e as proteínas de transporte de metais de transição (transferrina e ceruloplasmina), além de compostos obtidos diretamente da dieta, tais como vitaminas ( $\mathrm{C}, \mathrm{E}$ e betacaroteno) e minerais antioxidantes (zinco, cobre e selênio) (Bianchi et al., 2009).

Alguns autores também classificam os antioxidantes em intracelulares e extracelulares. Nesse contexto, enzimas como SOD e CAT são exemplos de agentes enzimáticos intracelulares, as quais convertem seus substratos, ânion superóxido e peróxido de hidrogênio, respectivamente, para formas menos reativas. Como antioxidantes extracelulares estão à albumina, a bilirrubina e o urato (Bianchi et al., 2009; Mekki et al., 2010; Nihi et al., 2010).

A enzima SOD corresponde a uma família de proteínas com diferentes grupos prostéticos em sua composição, e que constitui a primeira linha de defesa contra o excesso de oxidantes. Nas células eucarióticas, existem duas formas de SOD: SOD dependente de manganês (SOD-Mn), presente na mitocôndria; e a SOD citosólica dependente dos íons zinco e cobre (SOD$\mathrm{Zn} / \mathrm{Cu}$ ). Nos dois casos, a enzima catalisa a dismutação do superóxido em oxigênio e peróxido de hidrogênio. Umas das funções mais importantes da enzima SOD é impedir a formação do ânion superóxido, já que este último pode reagir com o óxido nítrico, resultando na formação de peroxinitrito (Droge, 2002).

O óxido nítrico é sintetizado a partir da L-arginina por ação da óxido nítrico sintetase (NOS), enzima presente no endotélio e nos macrófagos. Promove relaxamento do endotélio vascular (vasodilatação com redução da resistência periférica), inibe a agregação plaquetária e desempenha importante papel na síndrome de lesão por isquemia-reperfusão. Ele tem meia vida muito curta, em torno de milésimos de segundo, podendo rapidamente se converter em radical livre (RL) com efeito vasoconstritor, que pode ser anulado pela presença de vitamina C, que recupera o óxido nítrico. Além da situação de reperfusão pós-isquemia, a superprodução de óxido nítrico por macrófagos durante a inflamação tem sido implicada no choque séptico e em outros processos crônicos relacionados à presença de macrófagos ativados. Entre suas principais funções destacam-se a regulação da pressão arterial e a sinalização intercelular. Seu efeito tóxico, como radical livre, contribui de modo importante para a lesão tecidual que ocorre nos processos inflamatórios crônicos e na sepse, devido a sua interação com o radical superóxido, formando o peroxinitrito, substância altamente reativa (Sarni \& Leite, 2003).

A quantificação de radicais livres produzidos in vivo é difícil, pois eles têm alta reatividade e meia-vida curta (Bianchi et al., 2009; Emokpae et al., 2010; Gosmanova et al., 2011). Por essa razão diferentes marcadores têm sido utilizados em estudos que buscam avaliar o estresse oxidativo, tais como compostos relacionados com a peroxidação lipídica e proteica. Nesse sentido, vários estudos demonstraram aumento de estresse oxidativo em pacientes com DRC evidenciado pelo acúmulo de compostos reativos carbonílicos como marcadores elevados de peroxidação proteica e aumento das concentrações de 
malondialdeído (MDA) no plasma, como marcador de peroxidação lipídica elevada (Bianchi et al., 2009; Emokpae et al., 2010; Gosmanova \& Le, 2011). Vários estudos mostraram que marcadores de estresse oxidativo estão aumentados na DRC (Bayes et al., 2006; Zwolinska et al., 2006; Valko et al., 2007). O estresse oxidativo e a inflamação são características constantes de doença renal avançada e desempenham um papel importante na deterioração progressiva da função e estrutura renal, e estão associados a alguns fatores de risco cardiovascular e inúmeras outras complicações da DRC (Kao et al., 2010).

Além das alterações em marcadores de estresse oxidativo, pacientes renais crônicos podem apresentar mecanismos de defesa suprimidos, redução do sistema enzimático de defesa, bem como do sistema não enzimático favorecendo o ataque das EROs às células com consequente dano celular (Annuk et al., 2001; Cachofeiro et al., 2008). De fato, Bianchi et al. (2009) demonstraram em pacientes em hemodiálise aumento na peroxidação lipídica no plasma e diminuição na atividade da SOD, alterações que parecem contribuir para a ocorrência de distúrbios oxidativos.

Alguns estudos procuram fundamentar as alterações na atividade da SOD no fato de que muitas células em resposta ao estresse oxidativo estimulam a produção de enzimas antioxidantes, o que parece ocorrer em função de sua necessidade por tecidos específicos. Provavelmente, a atividade da SOD esteja elevada em indivíduos com DRC em função de sua maior demanda, por tratar-se de primeira enzima de defesa antioxidante (Oberg et al., 2004; Kalantar-Zadeh et al., 2005, Bianchi et al, 2009; Knap et al., 2009).

Segundo Knap et al. (2009), entre os fatores que poderiam desempenhar papel importante nas alterações da atividade da SOD em doentes renais estão a deficiência de oligoelementos essenciais para sua ativação, como o zinco, ou o aumento na produção do ânion superóxido e peróxido de hidrogênio encontrada na uremia e ainda a exposição aos elementos tais como o alumínio, o silício e ferro, durante a diálise. Todos esses fatores podem contribuir para redução da atividade da SOD.

Destaca-se que as interações entre as membranas de diálise e os neutrófilos no sangue podem ativar a produção de radicais livres, tais como ânion superóxido e peróxido de hidrogênio. Essas substâncias contribuem para a oxidação de lipídios, proteínas e ácidos nucleicos (Malliaraki et al., 2003), outro aspecto importante relacionado à função dos leucócitos na ativação de produção de espécies reativas de oxigênio induzida pela hemodiálise (Noleto Magalhães et al., 2011). A hemorreatividade entre as membranas e a hemoincompatibilidade dos componentes do sistema de diálise são considerados os principais fatores envolvidos na formação de excessivos radicais livres, fatores que podem contribuir para uma resposta positiva ao estresse oxidativo acarretando a ativação do sistema antioxidante (Morena et al., 2005).

Doentes renais crônicos são susceptíveis a desordens no metabolismo de lipídios e, consequentemente, a um perfil lipídico aterogênico. Estudos têm demonstrado relação entre o desenvolvimento de aterosclerose e o aumento da peroxidação lipídica na DRC por maior suscetibilidade às modificações oxidativas das LDL, com formação de dienos conjugados e produtos desta oxidação (Menevse et al., 2006; Negane et al., 2013). Ademais, as LDL são suscetíveis ao dano induzido por EROs e, quando oxidadas, parecem ser um fator importante para o desenvolvimento de lesões ateroscleróticas, promovendo dano direto e destruição da célula endotelial (Shivashekar et al., 2012; Negamma et al., 2014).

\section{Conclusão}

A análise realizada evidenciou que os portadores de doença renal crônica apresentam inúmeras alterações metabólicas que cursam com deficiências nutricionais importantes tanto de macronutrientes como de micronutrientes, sendo necessário o constante acompanhamento do estado nutricional por ser um forte preditor de morbidades e mortalidades. É consenso na literatura que uma combinação de marcadores nutricionais deve ser empregada para melhorar a precisão do diagnóstico nutricional. As limitações associadas à influência da DRC sobre os marcadores nutricionais, bem como aquelas decorrentes da falta de padrões de referência e de definição de pontos de corte associados ao risco, fazem com que o acompanhamento 
periódico seja a melhor forma de detectar as anormalidades no estado nutricional e de avaliar o impacto das intervenções. Ademias, pode-se constatar que o processo inflamatório relacionado à própria doença renal crônica está associado a uma gama de fatores durante o curso da doença, que contribuem para o aumento do estresse oxidativo e diminuição da atividade antioxidante, que irão culminar na maior causa de mortalidade nesses indivíduos portadores de DRC.

\section{Referências}

Abensur, H. (2010). Deficiência de ferro na doença renal crônica. Revista Brasileira de Hematologia e Hemoterapia, 32(2), 95-98. Recuperado em 24 junho, 2021, de https://doi.org/10.1590/S1516-84842010005000047.

Annuk M., Zilmer M., Lind L., Linde T., Fellström B. (2001). Oxidative stress and endothelial function in chronic renal failure. J Am Soc Nephrol, 12(12) 2747-2752.

Azevedo, Z. M. A., Silva, D. R., Dutra, M. V. P., Elsas, M. I. C., Barbosa-Silva, G. M. C. G., Fonseca, V. M. (2007). Associação entre ângulo de fase, PRISM I e gravidade da sepse. Revista Brasileira de Terapia Intensiva, 19(3) 297-303. https://doi.org/10.1590/S0103-507X2007000300005.

Barbosa-Silva, M. C., Barros, A. J., Post, C. L., Waitzberg, D. L., Heymsfield, S. B. (2003). Can bioelectrical impedance analysis identify malnutrition in preoperative nutrition assessment? Nutrition, 19(5) 422-426.

Barbosa-Silva, M. C., Barros, A. J., Wang, J., Heymsfield, S. B., Pierson, R. N. Jr. (2005). Bioelectrical impedance analysis: population reference values for phase angle by age and sex. Am J Clin Nutr, 82(1) 49-52.

Barreto, S. M. I., Avesani, C. M., Vale, B., Lemos, C., Bregman, R. (2008). Agreement between anthropometry and bioelectrical impedance for measuring body fat in nonobese and obese nondialyzed chronic kidney disease patients. J Ren Nutr, 18(4) 355-362.

Bastos, M. G., Bregman, R., Kirsztajn, G. M. (2010). Doença renal crônica: frequente e grave, mas também prevenível e tratável. Revista da Associação Médica Brasileira, 56(2) 248-253. https://doi.org/10.1590/S0104-42302010000200028.

Bayés, B., Pastor, M. C., Bonal, J., Foraster, A., Romero, R. (2006). Oxidative stress, inflammation and cardiovascular mortality in haemodialysis--role of seniority and intravenous ferrotherapy: analysis at 4 years of follow-up. Nephrol Dial Transplant, 21(4) 984-990.

Berbel, M. N., Pinto, M. P. R., Ponce, D., Balbi, A. L. (2011). Aspectos nutricionais na lesão renal aguda. Revista da Associação Médica Brasileira, 57(5) 600606. https://doi.org/10.1590/S0104-42302011000500022.

Bianchi, P. D., Barp, J., Thomé, F. S., Belló-Klein, A. (2009). Efeito de uma sessão de hemodiálise sobre o estresse oxidativo sistêmico de pacientes renais crônicos terminais. Braz. J. Nephrol, 31(3) 175-182.

Cabral, P. C., Diniz, A. S., Arruda, I. K. G. (2005). Avaliação nutricional de pacientes em hemodiálise. Revista de Nutrição, 18(1) 29-40.

Cachofeiro, V., Goicochea, M., Vinuesa, S. G., Oubiña, P., Lahera, V., Luño, J. (2008). Oxidative stress and inflammation, a link between chronic kidney disease and cardiovascular disease. Kidney International Supplements, 74 4-9.

Calado, I. L., Silva, A. A. M., França, A. K. T. C., Santos, A. M., Filho, N. S. (2009). Diagnóstico nutricional de pacientes em hemodiálise na cidade de São Luís (MA). Revista de Nutrição, 22(5) 687-696.

Calado, I. L., França, A. K. T. C., Santos, A. M., Filho, N. S. (2007). Avaliação Nutricional de Pacientes Renais em Programa de Hemodiálise em um Hospital Universitário de São Luís do Maranhão. Jornal Brasileiro de Nefrologia, 29(4).

Cassini, A. V., Malagutti, W., Rodrigues, F. S. M., Deus, R. B., Barnabe, A. S., Francisco, L., Ferraz, R. R. N. (2010). Avaliação dos principais fatores etiológicos em indivíduos portadores de insuficiência renal crônica em hemodiálise. ConScientiae Saúde, 9(3) 462-468.

Cesarino, C. B., Borges, P. P., Ribeiro, R. C. H. M., Ribeiro, D. F., Kusumota, L. (2013). Avaliação do risco cardiovascular de pacientes renais crônicos segundo critérios de Framingham. Acta Paulista de Enfermagem, 26(1) 101-107.

Costa, J. A. C., Vieira-Neto, O. M., Moysés N. M. (2003). Insuficiência renal aguda. Medicina, Faculdade de Medicina, Ribeirão Preto, 36 307-324.

Chaves, G. A., Graça, M. D., Gallo, V. C. (2007). Consumo alimentar e estado nutricional de pacientes com doença renal crônica em tratamento dialítico. Revista Brasileira de Ciências da Saúde, 3(14). http://seer.uscs.edu.br/index.php/revista_ciencias_saude.

Cuppari, L., Kamimura, M. A. (2009). Avaliação nutricional na doença renal crônica: desafios na prática clínica. Jornal Brasileiro de Nefrologia, 31 28-35.

Dantas, A. S., Martins, C., Lauria, E., Savatin, V. C., Coutinho, V., Liberali, R. (2012). Alterações bioquímicas e de conhecimento nutricional para controle da hiperfosfatemia e hiperpotassemia de pacientes em hemodiálise em Brasília. Revista Brasileira de Nutrição Clínica, 27(1) 17-23.

Luis, D. A., Aller, R., Bachiller, P., Sagrado, M. G., Martin J., Izaola O. (2007). Influence of hormonal status and oral intake on phase angle in HIV-infected men. Nutrition, 20(9) 731-734.

Draczevski, L., Teixeira, M. L. (2011). Avaliação do perfil bioquímico e parâmetros hematológicos em pacientes submetidos à hemodiálise. Revista Saúde e Pesquisa, 4(1) 15-22. http://www.cesumar.br/pesquisa/periodicos/index.php/saudpesq.

Droge, W. (2002). Free radicals in the physiological controlo f cell function. Physiological Reviews, 82 47-95. 
Eickemberg, M., Oliveira, C. C., Carneiro, R. A. K., Sampaio, L. R. (2011). Bioimpedância elétrica e sua aplicação em avaliação nutricional. Revista de Nutrição, 24(6) 883-893.

Emokpae, M. A., Uadia, P. O., Gadzama, A. A. (2010). Correlation of oxidative stress and inflammatory markers with the severity of sickle cell nephropathy. Annals of African Medicine, 9 141-146.

European Guidelines for the Nutritional Care of Adult Renal Patients European. (2002). Dietitians Special Interest Group of the EDTNA/ERCA. http://www.era-edta.org.

Gómez, J. M. L. (2011). Evolución y aplicaciones de la bioimpedância en el manejo de la enfermedad renal crônica. Nefrologia, 31(6) 630-634.

Gosmanova, E. O., Ngoc-Anh, L. E. (2011). Cardiovascular Complications in CKD Patients: Role of Oxidative Stress. Cardiology Research and Practice, 11.

Gupta, D., Lammersfeld, C. A., Burrows, J. L., Dahlk, S. L., Vashi, P. G., Grutsch, J. F., Hoffman, S., Lis, C. G. (2004). Bioelectrical impedance phase angle in clinical practice: implications for prognosis in advanced colorectal cancer. American Journal of Clinical Nutrition, 80(6) 134-138.

Gupta, D., Lammersfeld, C. A., Vashi, P. G., King, J., Dahlk, S. L., Grutsch, J. F., Lis, C. G. (2009). Bioelectrical impedance phase angle as a prognostic indicator in breast cancer. BMC Cancer, 8(1) 249.

Herselman, M., Esau, N., Kruger, J.-M., Labadarios, D., Moosa, M. R. (2010). Relationship between serum protein and mortality in adults on long-term hemodialysis: exhaustive review and meta-analysis. Nutrition, 26(1) 10-32. http://www.nutritionjrnl.com.

Filho, J. C. J., Cyrino, E. S., Gurjão, A. L. D., Braz, I. A., Gonçalves, R., Gobbi, S. (2010). Estimativa da composição corporal e análise de concordância entre analisadores de impedância bioelétrica bipolar e tetrapolar. Revista Brasileira de Medicina do Esporte, 16(1) 13-7.

Junior, A. L. R., Neto, O. P. A., Soares, T. A. M., Cunha, C. M., Pedrosa, L. A. K. (2017). Suplementação com vitamina D em pacientes com doença renal crônica associada à hipovitaminose D. Arquivos de Ciências da Saúde, 24(3) 03-07. https://www.cienciasdasaude.famerp.br/index.php/racs/article/view/754.

Junior, G. B. S., Bentes, A. C. S. N., Daher, E. F., Matos, S. M. A. (2006). Obesidade e Doença Renal Crônica. Jornal Brasileiro de Nefrologia, 28(3).

Kalantar-Zadeh, K., Braglia, A., Chow, J., Kwon, O., Kuwae, N., Colman, S., Cockram, D. B., Kopple, J. D. (2005). An anti-inflammatory and antioxidant nutritional supplement for hypoalbuminemic hemodialysis patients: a pilot/feasibility study. Journal of Renal Nutrition, 15(3) 318-331.

Kao, M. P. C., Ang, D. S. C., Pall, A., Struthers, A. D. (2010). Oxidative stress in renal dysfunction: mechanisms, clinical sequelae and therapeutic options. Journal of Human Hypertension, 24 1-8.

Knap, B., Prezelj, M., Buturović-Ponikvar, J., Ponikvar, R., Bren, A. F. (2009). Antioxidant Enzymes Show Adaptation to Oxidative Stress in Athletes and Increased Stress in Hemodialysis Patients. Therapeutic Apheresis and Dialysis, 13(4) 300-305.

Koehnlein, E. A., Yamada, A. N., Giannasi, A. C. B. (2008). Avaliação do estudo nutricional de pacientes em hemodiálise. Acta Scientiarum. Health Science, 30(1) 65-71.

Koppe, L., Fouque, D., Kalantar-Zadeh, K. (2019). Kidney cachexia or protein-energy wasting in chronic kidney disease: facts and numbers. J Cachexia Sarcopenia Muscle. Jun;10(3):479-84.

Kyle, U. G., Bosaeus, I., Lorenzo, A. D., Deurenberg, P., Elia, M., Gómez, J. M., Heitmann, B. L., Kent-Smith, L., Melchior, J., Pirlich, M., Scharfetter, H., Schols, A. M. W. J., Pichard, C. (2004). Bioelectrical Impedance Analysis - Part I: Review of Principles and Methods. Clinical Nutrition, 23(5) 1226-1243.

Leal, V. D. O., Leite Júnior, M., Mafra, D. (2008). Acidose metabólica na doença renal crônica: abordagem nutricional. Revista de Nutrição, 21 93-103.

Mafra, D., Forage, N. E. (2006). O papel do tecido adiposo na doença renal crônica. Jornal Brasileiro de Nefrologia, 28(2).

Malliaraki, N., Mpliamplias, D., Kampa, M., Perakis, K., Margioris, A. N., Castanas, E. (2003). Total and corrected antioxidant capacity in hemodialyzed patients. BMC Nephrology, 4(4) 1471-2369.

Massi, Z. A., Stenvinkel, P., Drueke, T. B. (2009). The Role of Oxidative Stress in Chronic Kidney Disease. Seminars in Dialysis, 22(4) 405-408.

Mazairac, A. H. A., Wit G. A., Grooteman, M. P. C., Penne, E. L., Weerd N. C., Dorpel, M. A., Nubé, M. J., Lévesque, R., Wee, P. M. T., Bots, M. L., Blankestijn, P. J. (2011). A composite score of protein-energy nutritional status predicts mortality in hemodialysis patients no better than its individual components. Nephrology Dialysis Transplantation, 26(6) 1962-1967.

Mekki, K., Taleb, W., Bouzidi, N., Kaddous, A., Bouchenak, M. (2010). Effect of hemodialysis and peritoneal dialysis on redox status in chronic renal failure patients: a comparative study. Lipids in Health and Disease, 9(93) 1-7.

Menevse, E., Sivrikaya, A., Karagözoglu, E., Tiftik, A. M., Türk, S. (2006). Study of Elements, Antioxidants and Lipid Peroxidation in Hemodialysis Patients. Turkish Journal of Medical Sciences, 36(5) 279-284.

Morena, M., Delbosc S., Dupuy, A., Canaud B., Cristol J. (2005). Overproduction of reactive oxygen species in end-stage renal disease patients: A potential component of hemodialysis-associated inflammation. Hemodialysis International, 9 37-46.

Mushnick, R., Fein, P. A., Mittman, N., Goel, N., Chattopadhyay, J., Avram, M. M. (2003). Relatinship of bioelectrical impedance parameters to nutrition and survival in peritoneal dialysis patients. Kidney International, 64(87) 53-56.

Nagamma, T., Ahmed, S., Pai, A., Mohan, S., Chathurvedi, A., Singh, P. P. (2014). Evaluation of oxidative stress and antioxidant activity in pre and post hemodialysis in chronic renal failure patients from Western region of Nepal. Bangladesh Journal of Medical Science, 13(1). 
Nagane, N. S., Ganu, J. V., Jagtap, P. E. (2012). Effect of oxidative stress in pre and post hemodialysis in chronic renal failure patients. International Journal of Biological \& Medical Research, 3(1) 1335-1337.

Nagane1, N. S., Ganu, J.V., Jagtap, P.E. (2013). Study of oxidative stress in pre- and post-hemodialysis in chronic renal failure patients. Biomedical Research, 24(4) 498-502.

National kidney foundation /kdoqi. (2006). Clinical practice guidelines for chronic kidney disease: executive summary. http://www.kidney.org.

Nihi, M. M., Manfro, R. C., Martins, C., Suliman, M., Murayama, Y., Riella, M. C., Lindholm, B., Nascimento, M. M. (2010). Associação entre gordura corporal, inflamação e estresse oxidativo na hemodiálise. Jornal Brasileiro de Nefrolologia,32(1) 11-17.

Oberg, B. P., McMenamin, E., Lucas, F. L., McMonagle, E., Morrow, J., Ikizler, T. A., Himmelfarb, J. (2004). Increased prevalence of oxidant stress and inflammation in patients with moderate to severe chronic kidney disease. Kidney International, 65 1009-1016.

Oliveira, E.A., Zheng, R., Carter, C.E., Mak, R.H. (2019). Cachexia/protein energy wasting syndrome in CKD: causation and treatment. Semin Dial. Nov;32(6):493-9

Oliveira, C. M. C., Kubrusly, M., Mota, R. S., Silva, C. A. B., Oliveira, V. N. (2010a). Desnutrição na insuficiência renal crônica: qual o melhor método diagnóstico na prática clínica? Jornal Brasileiro de Nefrologia, 32(1) 57-70.

Oliveira, C. M. C., Kubrusly, M., Mota, R. S., Silva, C. A. B., Choukroun, G., Oliveira, V. N. (2010b). The Phase Angle and Mass Body Cell as Markers of Nutritional Status in Hemodialysis Patients. Journal of Renal Nutrition, 20(5) 314-320.

Oliveira, G. T. C., Andrade, E. I. G., Acurcio, F. A., Cherchiglia, M. L., Correia, M. I. T. D. (2012). Avaliação nutricional de pacientes submetidos à hemodiálise em centros de Belo Horizonte. Revista da Associação Médica Brasileira, 58(2) 240-247.

Passoni, C. M. S. (2005). Antropometria na prática clínica. Revista do Núcleo de Ciências Biológicas e da Saúde,1(2) 25-32.

Passos, V. M. A., Barreto, S. M., Lima-Costa, M. F. F. (2004). Detection of renal dysfunction based on serum creatinine levels in a Brazilian community: Bambuí Health and Agein Study. Brazilian Journal Of Medical and Biological Research, 36(3) 393-401.

Piccoli, A., Pillon, L., Dumler, F. (2002). Impedance Vector Distribution by Sex, Race, Body Mass Index, and Age in the United States: Standard Reference Intervals as Bivariate Z Scores. Nutrition, 18(2).

Pupim, L. B., Kent, P., Caglar, K., Shyr, Y., Hakim, R. M., Ikizler, T. A. (2002). Improvement in nutritional parameters after initiation of chronic hemodialysis. American Journal of Kidney Diseases, 40(1) 143-151.

Richard, M. J., Arnaud, J.,Jurkovitz, C., Hachache, T., Meftahi, H., Laporte, F., Foret, M., Favier, A., Cordonnier, D. (1991). Trace Elements and Lipid Peroxidation Abnormalities in Patients with Chronic Renal Failure. Nephron, 57(1) 10-15.

Santos, N. S. J., Draibe, S. A., Kamimura, M. A., Cuppari, L. (2004). Albumina sérica como marcador nutricional de pacientes em hemodiálise. Revista de Nutrição, 17(3) 339-349.

Sarni, R. S., Leite, H. P. (2003). Radicais livres, anti-oxidantes e nutrição. Revista Brasileira de Nutrição Clínica, 18(2) 87-94.

Sasatomi, Y., Ito, K., Abe, Y., Miyake, K., Ogahara, S., Nakashima, H., Saito, T. (2012). Association of Hypoalbuminemia with Severe Anemia in Patients with Diabetic Nephrosclerosis. Renal Failure, 34(2) 189-193.

Schmidt, R. J., Pellegrino, B., MacKay, K., Parravani, T., Mustafa, R., Hobbs, G. (2012). Prevalence and Predictive Value of Hypoalbuminemia in Appalachians with Chronic Kidney Disease. World Journal of Nephrology and Urology, 1(1) 8-15.

Silva, M. R. B., Barbosa, V. A., Sousa, B. S., Silva, A. M. D. (2009). Avaliação Nutricional e Consumo Alimentar de Pacientes com Insuficiência Renal Crônica. Revista Brasileira de Pesquisa em Saúde, 11(4) 39-48.

Stefanelli, C., Andreoti, F. D., Quesada, K. R., Detregiachi, C. R. P. (2010). Avaliação nutricional de pacientes em hemodiálise. Journal of the Health Sciences Institute, São Paulo, 28(3) 268-271.

Stratta P., Canavese, C., Marengo, M., Mesiano, P., Besso, L., Quaglia, M., Bergamo, D., Monga, G., Mazzucco, G., Ciccone, G. (2007). Risk management of renal biopsy: 1387 cases over 30 years in a single centre. European Journal of Clinical Investigation, 37(12) 954-963.

Talluri, A., Liedtke, R., Mohamed, E. I., Maiolo, C., Martinoli, R., Lorenzo, A. (2003). The application of body cell mass index for studying muscle mass changes in health and disease conditions. Acta Diabetologica, 40 286-289.

Terra, F. S., Costa, A. M. D. D., Figueiredo, E. T., Morais, A. M., Costa, M. D., Costa, R. D. (2010). As principais complicações apresentadas pelos pacientes renais crônicos durante as sessões de hemodiálise. Revista da Sociedade Brasileira de Clínica Médica, 8(3) $187-192$.

Valko, M., Leibfritz, D., Moncol, J., Cronin, M. T. D., Mazur, M., Telser, J. (2007). Free radicals and antioxidants in normal physiological functions and human disease. International Journal of Biochemistry \& Cell Biology, 39 44-84.

Vaziri, N. D., Norris, K. (2011). Lipid Disorders and Their Relevance to Outcomes in Chronic Kidney Disease. Blood Purification, 31 189-196.

Zwołińska, D., Grzeszczak, W., Szczepańska, M., Kiliś-Pstrusińska, K., Szprynger, K. (2006). Lipid peroxidation and antioxidant enzymes in children on maintenance dialysis. Pediatric Nephrology, 21 705-710. 